\title{
Rapamycin Down-Regulates Inducible Nitric Oxide Synthase by Inducing Proteasomal Degradation
}

\author{
Hye Kyoung JiN, ${ }^{a, \#}$ Seong Hoon Ahn, ${ }^{b, \#}$ Jong Woo Yoon, ${ }^{a}$ Jong Woo PARK, ${ }^{a}$ Eun Kyung LeE, ${ }^{a}$ \\ Jeong Soo Yoo, ${ }^{a}$ Jae Cheol LeE, ${ }^{a}$ Wahn Soo CHoI, ${ }^{c}$ and Jeung-Whan HaN ${ }^{*}, a$ \\ ${ }^{a}$ Department of Biochemistry and Molecular Biology, College of Pharmacy, Sungkyunkwan University; Suwon 440-746, \\ Korea: ${ }^{b}$ Division of Molecular \& Life Science, Hanyang University; Ansan 426-791, Korea: and ${ }^{c}$ College of Medicine \\ Konkuk University; Chungju 380-701, Korea.
}

Received January 5, 2009; accepted March 16, 2009; published online March 16, 2009

\begin{abstract}
We investigated the effect of rapamycin, a specific inhibitor of the mammalian serine/threonine kinase, mammalian target of rapamycin (mTOR), on the expression of inducible nitric oxide synthase (iNOS) in lipopolysaccharide (LPS)-stimulated RAW 264.7 cells. Pretreatment of cells with rapamycin significantly inhibited LPS-induced nitrite production and the expression of iNOS protein in a dose-dependent manner. However, LPS-induced mRNA expression of iNOS and its concomitant activation of nuclear factor (NF)- $\kappa B$ remained unchanged by rapamycin. Intriguingly, LPS-induced nitrite production and iNOS protein expression were partially blocked at nanomolar concentrations of rapamycin, whereas phosphorylation of both p70 S6 kinase and 4E-BP1 was completely abolished. The suppression of LPS-induced iNOS expression by rapamycin was reversed by the protease inhibitor lactacystin. Furthermore, rapamycin treatment stimulated 20S proteasome activity, which was slightly elevated by LPS. Taken together, our findings strongly suggest that rapamycin down-regulates LPS-induced iNOS protein expression via proteasomal activation, as well as through inhibition of the mTOR signaling pathway.
\end{abstract}

Key words rapamycin; nitric oxide synthase; p70 S6 kinase; 4E-binding protein; proteasome

Rapamycin, a macrolide antibiotic, has a potent anti-proliferative effect in multiple cell types and is currently used in kidney transplantation as a clinical immunosuppressant. ${ }^{1)}$ Rapamycin interacts with FK506-binding protein $(12 \mathrm{kDa}$, FKBP12), and this complex inhibits the mammalian target of rapamycin (mTOR). Recent findings revealed that mTOR controls a diverse set of downstream effectors, some of which are important for cell growth, such as p70 S6 kinase and eukaryotic translation initiation factor 4E-binding proteins. ${ }^{2,3)}$ It is now believed that p70 S6 kinase participates in regulation of transcription, as well as translation of mRNAs required for the $\mathrm{G}_{1} / \mathrm{S}$ phase transition in the cell cycle. ${ }^{3)}$

Macrophage activation by lipopolysaccharide (LPS) is regulated by various mechanisms, including the phosphatidylinositol 3 (PI3)-kinase signaling pathway. This pathway has also been implicated in LPS-mediated activation of human monocytes. ${ }^{4)}$ p70 S6 kinase may be one downstream effector of PI3-kinase, and is also thought to be involved in production of nitric oxide (NO) and expression of inducible $\mathrm{NO}$ synthase (iNOS) in LPS-activated murine macrophages. ${ }^{5)}$ The precise mechanism of this signaling pathway is unclear. In general, iNOS expression is regulated at the transcriptional level through activation of several transcription factors, such as nuclear factor (NF)- $\kappa \mathrm{B}$, signal transducer and activator of transcription 1 (STAT1), and interferon regulatory factor 1 (IRF-1). These transcription factors bind to the promoter region of the iNOS gene, ${ }^{6-8)}$ thereby inducing iNOS protein expression and NO production. ${ }^{6,7,9)}$ iNOS expression and NO production lead to the activation of macrophages in response to pro-inflammatory cytokines and bacterial products. $^{9-11)}$

In this study, we examined the effect of rapamycin, a specific inhibitor of mTOR, on the expression of iNOS in RAW 264.7 macrophages in response to LPS. We investigated the molecular mechanisms by which LPS induces iNOS expression through the p70 S6 kinase pathway. Our results show that rapamycin suppresses LPS-induced iNOS expression and subsequent NO production. This might be partly mediated by the inhibition of p70 S6 kinase activity at nanomolar concentrations of rapamycin, and the activation of $20 \mathrm{~S}$ proteasomal activity at higher concentrations. Therefore, our results provide novel insight into the dual function of rapamycin in the regulation of LPS-induced iNOS expression, which includes mTOR signaling and proteasome activation pathways.

\section{MATERIALS AND METHODS}

Cell Culture and Reagents The murine macrophage cell line RAW 264.7 from ATCC (American Type Culture Collection) was grown at $37^{\circ} \mathrm{C}$ humidified atmosphere containing $5 \% \mathrm{CO}_{2}$ in Dulbecco's Modified Eagle's Medium (DMEM, Invitrogen) supplemented with $10 \%$ fetal bovine serum (Hyclone Laboratories) and $100 \mathrm{U} / \mathrm{ml}$ penicillin and $100 \mu \mathrm{g} / \mathrm{ml}$ streptomycin (Invitrogen). Rapamycin and lipopolysaccharide (from Escherichia coli, 011:B4) were obtained from Sigma and lactacystin was from Calbiochem.

Measurement of Nitrite and Cell Viability Assay The amount of nitrite in cell culture supernatants was spectrophotometrically determined using the Griess reaction method as described previously. ${ }^{12,13)}$ The effect of rapamycin on the viability of cultured RAW 264.7 cells was measured using 3-(4,5-dimethyl-thiazol-2-yl)-2,5-diphenyl-tetrazolium bromide (MTT) solution as previously described. ${ }^{14)}$

RT-PCR and Northern Blot Analysis For reverse transcription-polymerase chain reaction (RT-PCR), total RNA was extracted from RAW 264.7 cells using TRIzol $^{\circledR}$ reagent (Invitrogen) according to the manufacturer's instructions. RT- 
PCR was carried out using a GeneAmp ${ }^{\circledR}$ RNA PCR kit (Perkin-Elmer) with $1 \mu \mathrm{g}$ of total RNA. The amplified PCR products were electrophoresed on $1.2 \%$ agarose gels and stained with $0.5 \mu \mathrm{g} / \mathrm{ml}$ of ethidium bromide solution. The primers for iNOS were 5'-GTG TTC CAC CAG GAG ATG TTG-3' and 5'-CTC CTG CCC ACT GAG TTC GTC-3'; and the primers for glyceraldehyde-3-phosphate dehydrogenase (GAPDH) were 5'-TGA TGA CAT CAA GAA GGT GGT GAA G-3' and 5'-TCC TTG GAG GCC ATG TAG GCC AT-3'. For Northern blot analysis, $20 \mu \mathrm{g}$ of total RNA was separated on $1.2 \%$ formaldehyde-agarose gels, and transferred to Nytran ${ }^{\circledR}$ immobilization membranes (Schleicher \& Schuell Bioscience). Blots were sequentially hybridized with $\left[{ }^{32} \mathrm{P}\right]$-labeled cDNA probes (iNOS and GAPDH) in QuikHyb ${ }^{\circledR}$ solution (Stratagene Inc.). The probes for iNOS and GAPDH were prepared using PCR products by RadPrime DNA labeling kit (Invitrogen). The radioactivity in the blots was determined using a FUJI BAS 2000 bioimaging analyzer (Fuji Photo Film Co.).

Western Blot Analysis RAW 264.7 cells were rinsed twice with $50 \mathrm{~mm}$ Tris- $\mathrm{HCl}$ buffer $(\mathrm{pH} 7.5)$ containing $120 \mathrm{~mm} \mathrm{NaCl}, 20 \mathrm{~mm} \mathrm{NaF}, 1 \mathrm{~mm}$ ethylenediaminetetraacetic acid (EDTA), $5 \mathrm{~mm}$ ethylene glycol bis(2-aminoehyl ether)$N, N, N^{\prime}, N^{\prime}$-tetraacetic acid (EGTA), $15 \mathrm{~mm}$ sodium pyrophosphate, $30 \mathrm{~mm}$-nitrophenyl phosphate, $1 \mathrm{~mm}$ benzamidine, and $0.1 \mathrm{~mm}$ phenylmethylsulfonyl fluoride and then lysed in the same buffer containing 1\% Nonidet P-40. Cell lysates were cleared and prepared by centrifugation at $4{ }^{\circ} \mathrm{C}$ for $15 \mathrm{~min}$ at $12000 \times \boldsymbol{g}$. Thirty micrograms of each total protein was subjected to sodium dodecyl sulfate-polyacrylamide gel electrophoresis (SDS-PAGE) and Western blot analysis as described previously ${ }^{15}$ using anti-iNOS (Transduction Laboratories), anti-tubulin (Santa Cruz Technologies), anti-p $70^{\mathrm{S} 6 \mathrm{~K}}$ (Santa Cruz Technologies), and anti-4E-BP1 (Cell Signaling Technologies) antibodies.

Electrophoretic Mobility Shift Assay (EMSA) RAW 264.7 cells were pretreated with rapamycin followed by LPS ( $1 \mu \mathrm{g} / \mathrm{ml})$ stimulation for $2 \mathrm{~h}$. Nuclear proteins were then extracted and analyzed by EMSA as described previously. ${ }^{13)}$ The probe sequences contained the NF- $\kappa$ B consensus sequences as follows: NF- $\kappa \mathrm{BU}, 5^{\prime}-\mathrm{AGC}$ TTG GGG ACT TTC C-3' and NF- $\kappa$ BL, $3^{\prime}-\mathrm{CCC}$ CTG AAA GGT CGG C-5'. ${ }^{16}$ ) DNA-protein complexes were separated from unbound probes on native $6 \%$ PAGE and detected with a FUJI BAS 2000 bioimaging analyzer (Fuji Photo Film Co.).

20S Proteasome Activity Assay After stimulation of RAW 264.7 cells with $1 \mu \mathrm{g} / \mathrm{ml}$ of LPS for $18 \mathrm{~h}$, cells were treated with rapamycin $(5 \mu \mathrm{M})$ for an additional $6 \mathrm{~h}$ in the absence or presence of the proteasome inhibitor lactacystin $(10 \mu \mathrm{M})$. The soluble cell extracts $(0.5-2.0 \mathrm{mg}$ protein) were loaded onto Superose 6 (HR 10/30) FPLC columns equilibrated with $20 \mathrm{~mm}$ Tris/ $\mathrm{HCl}$ buffer $(\mathrm{pH} 7.5)$ containing $10 \%$ glycerol, $5 \mathrm{~mm}$ ATP, and $100 \mathrm{~mm} \mathrm{NaCl}$. The fractions $(0.5 \mathrm{ml}$ each) were analyzed using the proteasome activity assay kit (Chemicon).

\section{RESULTS}

Rapamycin Inhibits Nitrite Production in a Dose-Dependent Manner It has been reported that activation of the $\mathrm{mTOR} / \mathrm{p} 70$ S6 kinase pathway is involved in LPS-mediated
A

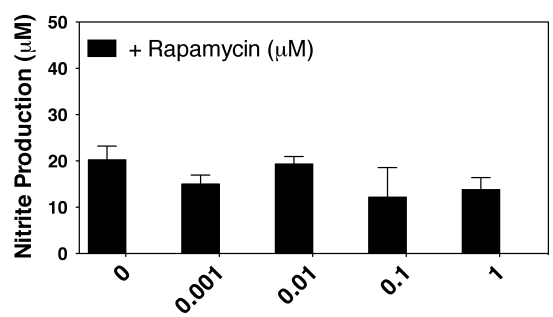

B

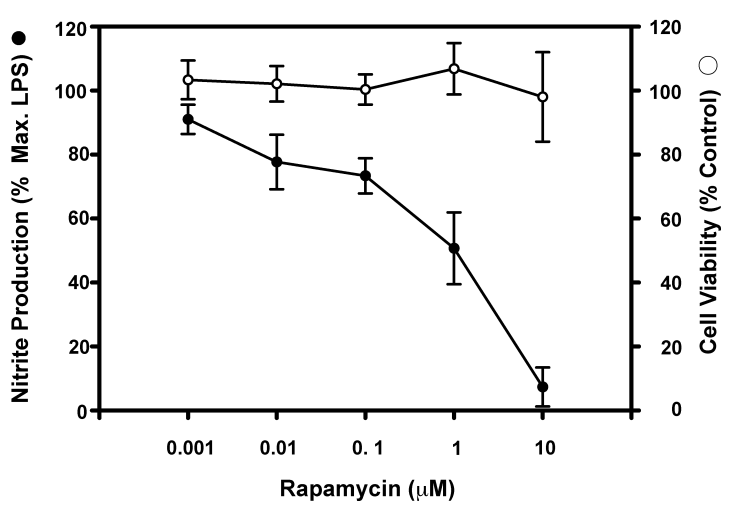

Fig. 1. Rapamycin Inhibits LPS-Induced Nitrite Production

(A) RAW 264.7 cells were treated with the indicated concentrations of rapamycin for $24 \mathrm{~h}$ and nitrite concentrations in culture media were determined using the Griess reaction. Values represent mean \pm S.E. from two separate experiments with triplicate determinations (B) RAW 264.7 cells were pretreated with the indicated concentrations of rapamycin for $30 \mathrm{~min}$ and then stimulated with $1 \mu \mathrm{g} / \mathrm{ml}$ LPS for $24 \mathrm{~h}$. Nitrite concentrations in culture media (-) were determined as in (A). Values represent mean \pm S.E. from four separate experiments with duplicate determinations. Nitrite concentrations in non-stimulated and LPS-stimulated RAW 264.7 cells were $22.19 \pm 0.45$ and $47.18 \pm$ $0.59 \mu \mathrm{M}$, respectively. Y-axis values mean the amount of nitrite induced by LPS. Cell viability (O) was determined using an MTT assay and expressed as the percentage of positive control (LPS stimulation) without the addition of rapamycin.

expression of iNOS and the subsequent production of NO in macrophages. ${ }^{5)}$ However, the mechanisms that regulate the turnover of the iNOS protein in response to LPS remain unknown. To examine the role of mTOR in LPS-induced nitrite production, we used the specific inhibitor, rapamycin. We first examined the amount of nitrite in non-stimulated RAW 264.7 cells by treatment with rapamycin $(0.001-1 \mu \mathrm{M})$ to exclude the possibility that rapamycin might affect the nitrite concentration produced from macrophage endothelial NOS (eNOS). The overall nitrite concentration in cells treated with rapamycin was not changed compared with that in nontreated cells (Fig. 1A). We then pretreated RAW 264.7 cells with rapamycin over a range of concentrations $(0.001-$ $10 \mu \mathrm{M})$ for $30 \mathrm{~min}$ and then stimulated with LPS $(1 \mu \mathrm{g} / \mathrm{ml})$ for $24 \mathrm{~h}$. LPS treatment increased nitrite production in RAW 264.7 cells approximately two-fold compared with untreated cells (data not shown). Pretreatment of cells with rapamycin inhibited LPS-induced nitrite production in a dose-dependent manner (Fig. 1B). Ten micromolar of rapamycin inhibited almost $100 \%$ of nitrite production compared to control cells stimulated with LPS. Interestingly, pretreatment of cells with nanomolar concentrations of rapamycin, which is sufficient for complete inhibition of p70 S6 kinase activity, ${ }^{17}$ resulted in a slight decrease $(<30 \%)$ in nitrite production. A cell viability test was carried out to determine if the inhibitory effect of rapamycin on nitrite production was attributable to nonspecific cytotoxicity. As shown in Fig. 1B, cell viability was 

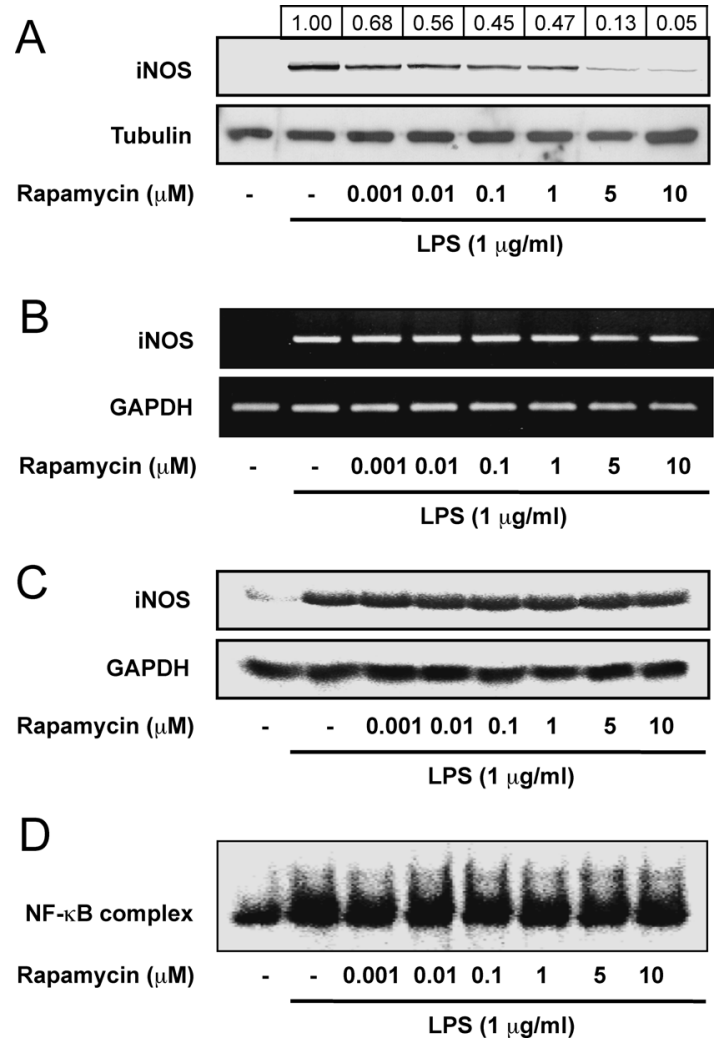

Fig. 2. Rapamycin Down-Regulates iNOS Protein

RAW 264.7 cells were preincubated with the indicated concentrations of rapamycin for $30 \mathrm{~min}$ and then treated with $1 \mu \mathrm{g} / \mathrm{ml}$ LPS for $20 \mathrm{~h}$. (A) iNOS protein expression was analyzed by Western blot using anti-iNOS antibody with tubulin as a reference The normalized values of iNOS protein expression over that of tubulin are indicated at the top of the Fig. 2A. (B) Total RNA was isolated using Trizol reagent, reverse-transcribed to cDNA by RT, and amplified by PCR using specific primers for murine iNOS and rat GAPDH as described in Materials and Methods. (C) Total RNA (20 $\mu \mathrm{g})$ was analyzed by Northern blot using probes for iNOS and GAPDH. (D) Cells were preincubated with the indicated concentrations of rapamycin for $30 \mathrm{~min}$ and then stimulated with LPS $(1 \mu \mathrm{g} / \mathrm{ml})$ for $2 \mathrm{~h}$. Nuclear extracts were prepared and subjected to EMSA as described in Materials and Methods.

not affected by treatment with rapamycin at any concentration compared to control cells stimulated with LPS, indicating that rapamycin suppresses LPS-induced nitrite production without cytotoxicity.

Rapamycin Reduces the Level of LPS-Induced iNOS Protein To investigate the molecular mechanism by which rapamycin inhibits the production of nitrite in response to LPS, we examined the changes in iNOS expression following rapamycin treatment. As shown in Fig. 2A, LPS stimulation of RAW 264.7 cells resulted in a dramatic increase in iNOS protein expression, whereas pretreatment with rapamycin inhibited LPS-induced iNOS expression in a dose-dependent manner. This finding is consistent with previous results in which rapamycin suppressed LPS-induced nitrite production (Fig. 1B). However, in contrast to the dose-dependent inhibition of iNOS protein expression by rapamycin, no significant changes in the level of iNOS mRNA production were observed following rapamycin pretreatment. This suggests that suppression of iNOS protein expression by rapamycin might be controlled at the translational and/or post-translational level rather than transcriptional level (Figs. 2B, C). To further confirm that the inhibition of iNOS expression was not transcriptionally regulated by rapamycin, we examined the activity of $\mathrm{NF}-\kappa \mathrm{B}$ in rapamycin treated cells. NF- $\kappa \mathrm{B}$ is a critical

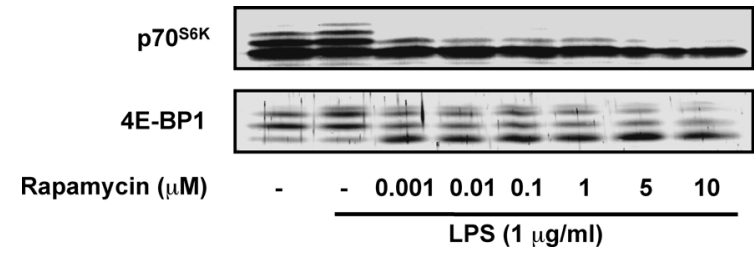

Fig. 3. Rapamycin Inhibits LPS-Induced Activation of p70 S6 Kinase and 4E-BP1 at Nanomolar Concentrations

RAW 264.7 cells were pretreated with the indicated concentrations of rapamycin for $30 \mathrm{~min}$ and then stimulated with LPS $(1 \mu \mathrm{g} / \mathrm{ml})$ for $24 \mathrm{~h}$ (for p70 S6 kinase analysis) or $3 \mathrm{~h}$ (for 4E-BP1 analysis). Cell extracts were then subjected to SDS-PAGE and Western blot analysis using anti-p70 S6 kinase and anti-4E-BP1 antibodies.

transcription factor known to regulate iNOS expression in response to stimulation by LPS or other inflammatory cytokines in RAW 264.7 cells. ${ }^{8}$ ) We hypothesized that if rapamycin influences iNOS expression at the post-transcriptional level, then the interaction between NF- $\kappa \mathrm{B}$ and the iNOS promoter would not be affected by rapamycin treatment. To test this, nuclear proteins containing NF- $\kappa \mathrm{B}$ were prepared and DNA binding activities between NF- $\kappa \mathrm{B}$ and $\mathrm{NF}-\kappa \mathrm{B}$ binding sites in the iNOS promoter were assessed by electrophoretic mobility shift assay. In LPS-stimulated RAW 264.7 cells, the binding of NF- $\kappa \mathrm{B}$ with DNA elements of the $\mathrm{NF}-\kappa \mathrm{B}$ binding site was induced. However, rapamycin pretreatment did not affect LPS-induced NF- $\kappa$ B binding activity (Fig. 2D). Collectively, these results strongly suggest that rapamycin inhibits iNOS expression at the translational and/or post-translational level and subsequently reduces nitrite production in LPS-stimulated RAW 264.7 cells.

Activation of p70 S6 Kinase and 4E-BP1 by LPS Is Partially Involved in iNOS Expression Since we observed an inhibitory effect of rapamycin on iNOS expression and the production of nitrite in response to LPS (Figs. 1B, 2), we next examined changes in the phosphorylation/activation of the downstream effectors of mTOR, p70 S6 kinase and 4E-BP1. It has been reported that rapamycin inhibits translation initiation through inactivation of p70 S6 kinase and 4EBP1. ${ }^{18)}$ Treatment of RAW 264.7 cells with LPS resulted in phosphorylation/activation of p70 S6 kinase and 4EBP1 compared with untreated cells, as determined by electrophoretic mobility shift. The LPS-induced mobility retardations of p70 S6 kinase and 4E-BP1 were dramatically blocked by pretreatment with rapamycin in a dose-dependent manner (Fig. 3). However, it should be noted that although rapamycin pretreatment at nanomolar concentrations $(>1 \mathrm{~nm})$ could completely inhibit the phosphorylation of p70 S6 kinase and 4E-BP1, nitrite production and the expression of iNOS were only slightly reduced at the same concentrations (Figs. 1B, Fig. 2). These observations indicate that stimulation of iNOS expression by LPS and the subsequent production of nitrite are regulated, at least in part, by mTOR signaling pathways including p70 S6 kinase and 4E-BP1, and suggest the involvement of other rapamycin targets in this process.

iNOS Degradation Is Induced by Rapamycin-Sensitive Proteasome Activity In addition to inhibition of translation initiation, rapamycin has been reported to delay the $G_{1}$ to $\mathrm{S}$ phase transition of the cell cycle through down-regulation of cyclin D3 protein via its proteasomal degradation. ${ }^{19}$ ) To examine the contribution of proteasomal activity to the re- 

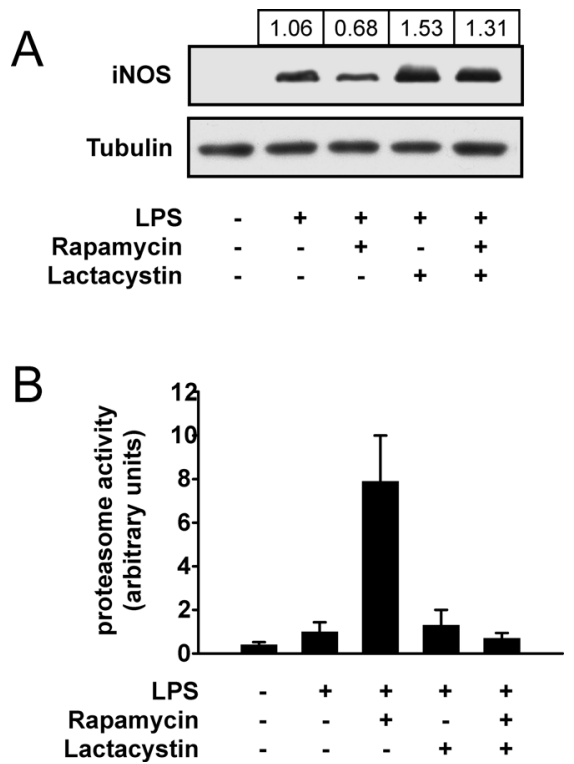

Fig. 4. Lactacystin Rescues iNOS Degradation Induced by Rapamycin

RAW 264.7 cells were stimulated with LPS $(1 \mu \mathrm{g} / \mathrm{ml})$ for $18 \mathrm{~h}$. Cells were then treated with rapamycin $(5 \mu \mathrm{M})$ for $6 \mathrm{~h}$ in the absence or presence of lactacystin $(10 \mu \mathrm{M})$. (A) Cells were harvested and the cell extracts were subjected to SDS-PAGE and Western blot analysis. The normalized values of iNOS protein expression over that of tubulin are indicated at the top of the Fig. 4A. (B) Cell extracts were centrifuged and fractionated using a Superose 6 (HR 10/30) FPLC column. The collected fractions were assayed for 20S proteasome activity as described in Materials and Methods. Values represent mean \pm S.E. from three independent experiments.

duction of iNOS expression in response to rapamycin treatment, we made use of the proteasome inhibitor lactacystin. Surprisingly, lactacystin pretreatment $(10 \mu \mathrm{M})$ for $6 \mathrm{~h}$ completely abrogated the effect of rapamycin LPS-stimulated RAW 264.7 cells, with little effect on cells treated with LPS alone (Fig. 4A). This result suggests that rapamycin regulation of nitrite production could be mediated by degradation of iNOS protein through proteasomal activation as well. To directly determine the influence of rapamycin on proteasome activity, we used the fluorogenic peptide Suc-Leu-Leu-ValTyr-7-amido-4-methylcoumarin as a $20 \mathrm{~S}$ proteasome substrate and assayed its degradation in the presence or absence of rapamycin. As expected, pretreatment of RAW 264.7 cells with rapamycin dramatically increased LPS-stimulated $20 \mathrm{~S}$ proteasome activity up to 16 -fold. This stimulation was completely abrogated by the addition of lactacystin (Fig. 4B). These results suggest that the reduction of iNOS expression at higher concentrations of rapamycin is mainly due to $20 \mathrm{~S}$ proteasome activation. Taken together, our findings show that rapamycin acts through two distinct mechanisms to regulate iNOS protein expression: inactivation of the mTOR downstream effectors p70 S6 kinase/4E-BP1 and activation of the $20 \mathrm{~S}$ proteasome.

\section{DISCUSSION}

A complete understanding of the cellular signaling mechanisms involved in the induction of iNOS could reveal novel targets for therapeutic intervention in NO-mediated inflammatory diseases. Earlier studies have shown that LPS activates multiple mitogen-activated protein kinase family members, such as p42/p44 MAPK, p46/p54 c-jun N-terminal kinase $(\mathrm{JNK}) /$ stress-activated protein kinase (SAPK), and p38
Hog in RAW 264.7 cells. $^{20,21)}$ Recently, several groups have demonstrated a critical role for the PI3-kinase pathway involving protein kinase $\mathrm{B}$ (PKB) and p70 S6 kinase in LPSmediated NO production. ${ }^{22,23)}$ However, the exact roles of these pathways on iNOS expression are still controversial. PI3-kinase has been shown to negatively regulate iNOS expression in LPS- or cytokine-stimulated C6 glial cells. ${ }^{24)}$ In addition, LPS-mediated NO production involves PI3-kinaseindependent pathways in RAW 264.7 cells. $^{25)}$ In particular, treatment of RAW 264.7 cells with rapamycin (from 10 to $200 \mathrm{~nm}$ ) does not affect NO synthesis in response to LPS, suggesting that the mTOR signaling pathway is not responsible for these effects. ${ }^{26)}$

These reports prompted us to study the exact role of the rapamycin-mediated signaling pathway in NO production and iNOS expression in response to LPS. In the present study, we found that LPS-induced NO production was inhibited by rapamycin in a dose-dependent manner without cytotoxicity, consistent with the reduction of iNOS protein expression. This result shows that the mTOR signal transduction pathway is involved in regulation of iNOS expression. Although most known iNOS inhibitors regulate iNOS expression at the transcriptional level, it has recently been reported that aspirin and sodium salicylate can also inhibit cytokine-induced NO production without affecting iNOS mRNA expression in RAW 264.7 cells. ${ }^{27)}$ In accordance with this report, we have shown here that iNOS protein production is suppressed by rapamycin n LPS-stimulated RAW 264.7 cells. This regulation appears to occur at the translational and/or post-translational level, since rapamycin had no effect on iNOS mRNA expression or NF- $\kappa \mathrm{B}$ binding to the iNOS promoter (Fig. 2).

It has been reported that phosphorylation of both p70 S6 kinase and 4E-BP1 requires kinase active mTOR, and that these phosphorylations are abolished by nanomolar concentrations of rapamycin. ${ }^{20,26,27)}$ In this study, LPS-induced phosphorylation of p70 S6 kinase and 4E-BP1 was completely blocked by $1 \mathrm{~nm}$ of rapamycin, whereas NO production was partially inhibited at the same concentration (Figs. 1B, 3). Complete inhibition of NO production required a 1000 -fold higher concentration of rapamycin $(10 \mu \mathrm{M})$ (Fig. 1B). On the other hand, the proteasome inhibitor lactacystin could completely rescue the down-regulation of iNOS protein caused by rapamycin. This suggests that rapamycin reduces the level of iNOS protein by promoting its degradation through $20 \mathrm{~S}$ proteasomal activation (Fig. 4). Consistent with our results, it has been shown that rapamycin decreases the half-life of cyclin D1 protein and that this is partially abrogated by the proteasome inhibitor $N$-acetyl-leucyl-leucyl-norleucinal. ${ }^{3,28)}$ Therefore, future studies are required to elucidate novel rapamycin-sensitive targets that are down-regulated by micromolar concentrations of rapamycin in LPS-stimulated RAW 264.7 cells. Taken together, our results suggest that LPS-induced iNOS expression by RAW 264.7 macrophages is regulated by a dual mechanism involving mTOR signaling and proteasomal activation pathways that are dependent on the concentration of rapamycin.

Acknowledgments This study was supported by Grant No. R01-1999-000-00127-0 from the Basic Research Program of the Korea Science and Engineering Foundation. 
S.H.A. was supported by the research fund of Hanyang University (HY-2005-N).

\section{REFERENCES}

1) Gingras A. C., Raught B., Sonenberg N., Genes Dev., 15, 807-826 (2001).

2) Stan R., McLaughlin M. M., Cafferkey R., Johnson R. K., Rosenberg M., Livi G. P., J. Biol. Chem., 269, 32027-32030 (1994).

3) Hashemolhosseini S., Nagamine Y., Morley S. J., Desrivieres S., Mercep L., Ferrari S., J. Biol. Chem., 273, 14424-14429 (1998).

4) Herrera-Velit P., Reiner N. E., J. Immunol., 156, $1157-1165$ (1996).

5) Seo J. Y., Lee J. H., Kim N. W., Kim Y. J., Chang S. H., Ko N. Y., Her E., Yoo Y. H., Kim J. W., Lee B. Y., Lee H. Y., Kim Y. M., Choi W. S., J. Pharm. Pharmacol., 57, 911-918 (2005).

6) Xie Q. W., Whisnant R., Nathan C., J. Exp. Med., 177, 1779-1784 (1993).

7) Lowenstein C. J., Alley E. W., Raval P., Snowman A. M., Snyder S. H., Russell S. W., Murphy W. J., Proc. Natl. Acad. Sci. U.S.A., 90, 97309734 (1993).

8) Xie Q. W., Kashiwabara Y., Nathan C., J. Biol. Chem., 269, 47054708 (1994).

9) MacMicking J., Xie Q. W., Nathan C., Annu. Rev. Immunol., 15, 323 350 (1997).

10) Nathan C., FASEB J., 6, 3051-3064 (1992).

11) Terenzi F., Diaz-Guerra M. J., Casado M., Hortelano S., Leoni S., Bosca L., J. Biol. Chem., 270, 6017-6021 (1995).

12) Green L. C., Wagner D. A., Glogowski J., Skipper P. L., Wishnok J. S., Tannenbaum S. R., Anal. Biochem., 126, 131-138 (1982).

13) Shin S. G., Kang J. K., Lee K. R., Lee H. W., Han J. W., Choi W. S., J. Toxicol. Environ. Health A, 68, 2119-2131 (2005).

14) Kim E. J., Jin H. K., Kim Y. K., Lee H. Y., Lee S. Y., Lee K. R., Zee
O. P., Han J. W., Lee H. W., Biochem. Pharmacol., 61, 903-910 (2001).

15) Yoon J. W., Kang J. K., Lee K. R., Lee H. W., Han J. W., Seo D. W., Kim Y. K., J. Toxicol. Environ. Health A, 68, 2005-2017 (2005).

16) Lee B. G., Kim S. H., Zee O. P., Lee K. R., Lee H. Y., Han J. W., Lee H. W., Eur. J. Pharmacol., 406, 301-309 (2000).

17) Han J. W., Pearson R. B., Dennis P. B., Thomas G., J. Biol. Chem., 270 21396-21403 (1995).

18) Chung J., Kuo C. J., Crabtree G. R., Blenis J., Cell, 69, 1227-1236 (1992).

19) Garcia-Morales P., Hernando E., Carrasco-Garcia E., MenendezGutierrez M. P., Saceda M., Martinez-Lacaci I., Mol. Cancer Ther., 5, 2172-2181 (2006)

20) Sanghera J. S., Weinstein S. L., Aluwalia M., Girn J., Pelech S. L., J. Immunol., 156, 4457-4465 (1996).

21) Denkers E. Y., Butcher B. A., Del Rio L., Kim L., Immunol. Rev., 201, 191-205 (2004).

22) Tzeng S. F., Huang H. Y., Lee T. I., Jwo J. K., J. Neurosci. Res., 81, 666-676 (2005)

23) Park S. J., Lee S. C., Hong S. H., Kim H. M., Cell Biol. Toxicol., 18, $121-130$ (2002).

24) Pahan K., Raymond J. R., Singh I., J. Biol. Chem., 274, 7528-7536 (1999).

25) Salh B., Wagey R., Marotta A., Tao J. S., Pelech S., J. Immunol., 161, 6947-6954 (1998)

26) Diaz-Guerra M. J., Castrillo A., Martin-Sanz P., Bosca L., J. Immunol., 162, 6184-6190 (1999).

27) Ryu Y. S., Lee J. H., Seok J. H., Hong J. H., Lee Y. S., Lim J. H., Kim Y. M., Hur G. M., Biochem. Biophys. Res. Commun., 272, 758-764 (2000).

28) Dong J., Peng J., Zhang H., Mondesire W. H., Jian W., Mills G. B., Hung M. C., Meric-Bernstam F., Cancer Res., 65, 1961-1972 (2005). 